\title{
Role of Intact Parathormone Levels as Early Predictor of Asymptomatic Hypocalcaemia after Total Thyroidectomy
}

\author{
D. Rajiv Raj ${ }^{1}$, Prabhu Purusothaman ${ }^{2}$
}

1Department of Surgery, Sri Ramachandra Institute of Higher Education and Research, Chennai, Tamilnadu, India.

2Department of Surgery, Sri Ramachandra Institute of Higher Education and Research, Chennai, Tamilnadu, India.

\section{ABSTRACT}

\section{BACKGROUND}

Asymptomatic hypocalcemia is one of the complications of total thyroidectomy. The symptoms of low calcium levels are sometimes evident only after about 48-72 hours after surgery. Serum calcium levels are not reliable in identifying these patients, because a large number of them are only those with transient hypocalcemia. This study was carried out to evaluate the role of intact parathormone levels in predicting asymptomatic hypocalcemia.

\section{METHODS}

This cohort study was carried out among 43 patients, who underwent total or completion thyroidectomy. The serum ionized calcium \& creatinine levels were measured preoperatively, to rule out pre-operative hypoparathyroidism. Following surgery (one-hour post operatively), the patients' serum PTH levels and serum ionized calcium levels were measured. Simultaneously, hypocalcemia was clinically evaluated.

\section{RESULTS}

Out of 43 patients, 30 patients had low hypocalcemia $(<1.1 \mathrm{mmol} / \mathrm{l})$ one hour postoperatively. The incidence of initial asymptomatic hypocalcaemia post-surgery was $70.5 \%$. All patients with low iPTH, except one, also had hypocalcaemia initially. The patient with normal calcium subsequently had hypocalcaemia at 6 hours.

\section{CONCLUSIONS}

With the prediction of asymptomatic hypocalcemia in the early post-operative period, it is possible to start timely treatment and thereby prevent the complications of hypocalcemia.

\section{KEY WORDS}

Asymptomatic Hypocalcaemia, Hypoparathyroidism, Ionized Calcium, Total Thyroidectomy
Corresponding Author: Dr. Prabhu Purusothaman, Department of Surgery, Sri Ramachandra Institute of Higher Education and Research, Chennai, Tamilnadu, India.

E-mail: prabbond@gmail.com

DOI: 10.14260/jemds/2020/162

Financial or Other Competing Interests: None.

How to Cite This Article:

Raj DR. Purusothaman P, Role of intact parathormone levels as early predictor of asymptomatic hypocalcaemia after total thyroidectomy. J. Evolution Med. Dent. Sci. 2020;9(10):745-748, DOI: 10.14260/jemds/2020/162

Submission 18-12-2019, Peer Review 08-02-2020, Acceptance 15-02-2020, Published 09-03-2020.

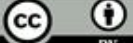




\section{BACKGROUND}

Total thyroidectomy is often indicated in thyroid malignancy and goiter. They are absolutely indicated when the thyroid nodules create compression effects like dysphagia, dyspnea, etc. A total thyroidectomy procedure is performed by dissecting both the lobes of thyroid and the parathyroid glands, in addition to dissection of the vulnerable lymph nodes. Although few surgeons advocate for parathyroid reimplantation, most patients suffer complications of PostSurgical Hypoparathyroidism (PoSH), characterized by transient hypocalcemia.[1] The incidence of hypocalcemia according to various studies are up to $46 \% .{ }^{[2]}$ The transient hypocalcemia occurring following thyroidectomy is often asymptomatic. It manifests within 48-72 hours and is often missed due to lack of any clinical presentations. The British Association of Endocrine and Thyroid Surgeons defines postoperative transient hypocalcemia as a calcium levels $<2.1$ $\mathrm{mmol} / \mathrm{L}$ on the day one post-operative period.[3] So far, there has been no consensus on any protocol for early detection of transient hypocalcemia. It has therefore been a routine procedure for the surgeon to wait for the features to manifest. Serum ionized calcium levels alone do not reliably identify these cases. A newer alternative for predicting transient hypocalcemia has been intact parathyroid hormones. Detection of these levels during the immediate post-operative period is considered as a reliable indicator for hypocalcemia. This is due to the fact that it has a half-life of 24 minutes.[4] Moreover, its physiological ability to rise along with vitamin $\mathrm{D}$ when there is a fall in calcium levels makes it a distinct predictor of hypocalcemia. It would therefore help the treating clinician to start the management of postoperative hypocalcemia early. There are very few studies published to evaluate the potential role of parathyroid levels in predicting transient hypocalcemia in post-thyroidectomy patients. The potential role of parathyroid levels can be best assessed in a prospective manner and can also indicate the impact of developing a standard protocol for detecting preventing complications of transient hypocalcemia.

This study was carried out to evaluate the role of intact parathyroid hormone levels in predicting transient hypocalcaemia following total thyroidectomy.

\section{METHODS}

This is a prospective cohort study carried out among all the patients who underwent total or completion thyroidectomy for any pathological indication in the Department of General Surgery of our tertiary teaching institution over a period of 18 months. A total of 43 patients participated in the study. All adults with clinical/pathological/surgical indication for total or completion thyroidectomy were included in the study. Patients with renal impairment/co-existing hyperparathyroidism were excluded.

\section{Ethical Approval and Informed Consent}

Approval was obtained from the Institutional Ethics Committee prior to the commencement of the study. Each participant was explained in detail about the study and informed consent was obtained prior to the data collection.

\section{Data Collection}

All participants underwent standard pre-operative assessment for total or completion thyroidectomy, including fine needle aspiration cytology, ultrasonography of the neck, thyroid function tests, neck x ray and vocal cord evaluation. In select cases, where retrosternal extension was suspected, CECT was done. The demographic details, indication for the surgery and the type of surgery (total or completion thyroidectomy). Serum creatinine levels to rule out renal dysfunction, and ionized calcium levels to exclude hyperparathyroidism were ascertained preoperatively. Serum intact parathyroid hormone (iPTH) levels and baseline serum ionized calcium levels were assessed at one hour following surgery (skin closure). The iPTH levels were measured using rapid assay, and results were obtained within 30 minutes. Clinical assessment of hypocalcemia was done by evaluating the symptoms and signs. A prophylaxis of calcium supplementation was initiated at four hours following the surgery ( $500 \mathrm{mg}$ ). Serum ionized calcium levels were measured every 6 hours to see if there is any response to calcium supplements. Once the ionized calcium levels were normalized, measurement was done daily until discharge. In patients with normal iPTH levels and even those with initial biochemical hypocalcemia, the patients were monitored, until discharge for symptoms of hypocalcemia without any oral calcium supplementation. Oral calcium supplementation was initiated only if clinical features of hypocalcaemia manifested.

\section{Data Analysis}

Data was entered and analyzed using SPSS ver. 21 software. The cut off values for iPTH levels were considered at $>15$ $\mathrm{pg} / \mathrm{ml}$. Percentages were computed for the intact parathyroid hormone levels. Chi square test was used to evaluate the statistical significance between the iPTH levels and hypocalcemia. A p value $<0.05$ was considered statistically significant. Validity indicators like specificity, positive and negative predictive values were used to evaluate the role of iPTH levels in predicting hypocalcemia.

\section{RESULTS}

This study was carried out among 43 patients who underwent total or completion thyroidectomy. Majority of the participants were aged between $40-50$ years $(32.5 \%)$ and were females (81.4\%). The most common indication for thyroidectomy was multinodular goiter (72.2\%). (table 1) Majority of the participants in this study had an elevated iPTH levels $>15 \mathrm{pg} / \mathrm{ml}$ (84\%). (figure 1) The incidence of transient hypocalcemia in this study was $69.7 \%$. We evaluated the statistical significance between iPTH levels and calcium levels. It was observed that participants with elevated iPTH levels had increased incidence of hypocalcemia (69.4\%) while those with normal iPTH levels also had increased incidence of hypocalcemia (71.4\%). The observed association was statistically not significant $\left(\chi^{2} 0.05=0.010\right)$. (table 2). Our study explored the validity of iPTH levels in predicting hypocalcemia. The validity parameters are given in 
table 3. The specificity of iPTH levels was $85 \%$ while the positive predictive value at one hour was $72 \%$ and the same at 6 hours was $100 \%$.

\begin{tabular}{|c|c|c|c|}
\hline Sl. No. & Characteristics & Frequency $(n=43)$ & Percentage (\%) \\
\hline \multirow{6}{*}{1} & \multicolumn{3}{|c|}{ Age (in Years) } \\
\hline & $<30$ & 6 & 13.9 \\
\hline & $30-40$ & 10 & 23.3 \\
\hline & $40-50$ & 14 & 32.5 \\
\hline & $50-60$ & 9 & 20.9 \\
\hline & $>60$ & 4 & 9.4 \\
\hline \multirow{3}{*}{2} & \multicolumn{3}{|c|}{$\begin{array}{l}\text { Sex } \\
\text { nex }\end{array}$} \\
\hline & Males & 8 & 18.6 \\
\hline & Females & 35 & 81.4 \\
\hline \multirow{7}{*}{3} & \multicolumn{3}{|c|}{ Indication for Thyroidectomy } \\
\hline & Multinodular goiter & 31 & 72.2 \\
\hline & Papillary carcinoma & 5 & 11.6 \\
\hline & Follicular carcinoma & 3 & 6.9 \\
\hline & Hashimoto's thyroiditis & 2 & 4.7 \\
\hline & Hurthle cell neoplasm & 1 & 2.3 \\
\hline & Colloid goiter & 1 & 2.3 \\
\hline
\end{tabular}

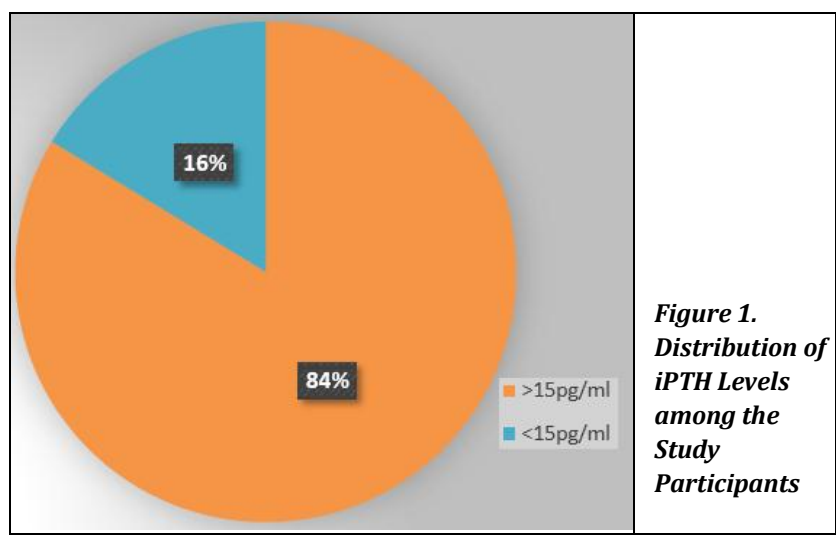

\begin{tabular}{|c|c|c|c|c|c|c|}
\hline Sl. & iPTH Levels & & Calci & um levels & Chi & \\
\hline No. & (pg/ml) & $\mathbf{N}$ & Hypocalcemia & Normocalcaemia & Sq. & $\mathbf{p}$ \\
\hline 1 & $>15$ & 36 & $25(69.4)$ & \begin{tabular}{|l|l}
$11(30.5)$ \\
\end{tabular} & & \\
\hline 2 & $<15$ & 7 & $5(71.4)$ & $2(28.6)$ & & \\
\hline
\end{tabular}

\begin{tabular}{|c|c|c|}
\hline Sl. No. & Validity Parameters & Percentage (\%) \\
\hline 1 & Specificity & $85 \%$ \\
\hline 2 & Positive predictive value & $72 \%$ \\
\hline 3 & Negative predictive value & $31 \%$ \\
\hline \multicolumn{3}{|c|}{ Positive predictive value after 6 hrs. } \\
\hline 4 & Table 3. Validity of iPTH in Detecting Hypocalcemia \\
\hline \multicolumn{3}{|c|}{} \\
\hline
\end{tabular}

\section{DISCUSSION}

The incidence of hypocalcemia post total/completion thyroidectomy is high. In most situations, this hypocalcaemia is only transient and does not warrant any monitoring or treatment. However, there are cases, few, though they may be, who do develop permanent hypocalcaemia. This becomes evident only later, sometimes as late as 72 hours, when the symptoms and signs of hypocalcaemia become apparent. The problem, therefore, presenting to the surgeon, is the difficulty in early identification of these cases. The issue is to differentiate, early in the postoperative cases, asymptomatic initial transient hypocalcaemia, from those at risk for prolonged or permanent hypocalcaemia. There are several studies regarding the management of hypocalcaemia following thyroid surgery. Some have never treated hypocalcaemia and waited till symptoms appear. Some prescribe calcium supplementation and Vitamin D routinely for all patients.[5-7]

Routine calcium supplementation and Vitamin D for all patients, is not without problems. Iatrogenic hypercalcemia, which it can lead to, is fraught with troubles. Unnecessary Vitamin D therapy can cause Vitamin D induced inhibition of residual parathyroid function which, in cases of temporary hypocalcaemia, may become permanent. ${ }^{[8]}$ On the other hand, therapy only when clinical features appear, has not been proven trustworthy. Symptoms manifest only 2-3 days postsurgery and at that point of time aggressive management is necessary, failing which dreadful complications have been reported.[9]

Intact parathyroid hormone (iPTH) assay, and its role as a predictor of such cases, has been extensively explored. Several studies have been done with iPTH measurement; preoperatively, intra-operatively, post operatively and even 6-12 hrs post operatively. Measurement at 6-12 hrs postsurgery has been found to have predictive value. However, a delayed identification of these patients would mean that the treatment is also delayed. Therefore, iPTH measured onehour post-surgery holds good, due to its short half-life.

All the patients in our study have also been screened for preexisting hypocalcemia by doing serum ionized calcium. Renal dysfunction was also ruled out prior to the selection. Intact parathyroid hormone as an early predictor of asymptomatic hypocalcaemia has been studied. It has been shown that low levels $(<15 \mathrm{pg} / \mathrm{ml})$ correlate well with prolonged low calcium levels. All, but two of the seven cases, had hypocalcaemia right from the first hour. All were hypocalcaemic at 6 hours. The experience of the surgeon, intraoperative anatomy and handling of the parathyroid glands leading to ischemia of the glands have not been taken into account as they are variable and operator dependent. There are adequate evidences to suggest that intra- operative manipulation does not correlate with post-operative calcium levels. ${ }^{[5,6]}$

In our study hypocalcemia occurred in nearly $69.7 \%$ of the patients ( 30 out of 43). iPTH levels were low in 7 of these 30 patients. These patients showed falling calcium levels and later normalized after calcium supplementation was started. None of the patients with normal iPTH levels, even those with initial hypocalcaemia, showed any clinical features of hypocalcaemia until discharge 3 to 4 days later. iPTH at one hour, therefore, has proved as an early indicator to differentiate between patients with asymptomatic initial hypocalcemia and patients who may have the probability of developing prolonged and symptomatic hypocalcaemia postsurgery.

As early intervention, oral calcium supplementation was started in 7 patients who had decreased iPTH, none developed symptoms of hypocalcaemia. Moreover, they did not progress to biochemical hypocalcaemia, as their serial ionized calcium levels increased after calcium supplementation. The study concluded with a specificity of $85 \%$, and positive predictive value of $72 \%$ and negative predictive value of $31 \%$ for iPTH. But the positive predictive 
value after $6 \mathrm{hrs}$ to predict hypocalcaemia was 100\% (table 3 ), which proves that it is an early predictor. It is also suggested that ionized calcium levels be measured only 6 hours postoperatively as the fall in ionized calcium levels has occurred significantly only after $6 \mathrm{hrs}$ in both the group of patients. Further study is needed to explore this aspect.

\section{CONCLUSIONS}

It can be said that iPTH measured post-operatively at 1 hour is a reliable indicator of predicting asymptomatic hypocalcemia post total/completion thyroidectomy. Further, the cost of doing an estimation of iPTH is significantly less than serial measurements of serum ionized calcium. Based on our study, routine post op management of total/completion thyroidectomy patients can be established using a standard protocol involving calcium supplementation. Thus, early management can be instituted before clinical features manifest and unnecessary monitoring of patients can be avoided.

\section{REFERENCES}

[1] Bhattacharyya N, Fried MP. Assessment of the morbidity and complications of total thyroidectomy. Arch Otorhinolaryngol Head Neck Surg 2002;128 (4):389-92.

[2] Edafe 0, Balasubramanian SP. Incidence, prevalence and risk factors for post-surgical hypocalcemia and hypothyroidism. Gland Surg 2017;6 (Suppl 1):S59-S68.
[3] Leiker AJ, Yen TWF, Eastwood DC, et al. Factors that influence parathyroid hormone half-life: determining if new intraoperative criteria are needed? JAMA Surg 2013;148 (7):602-6.

[4] Paul JM, Irvine R, Bugis S, et al. Intact parathyroid hormone measurement 1 hour after thyroid surgery identifies individuals at high risk for the development of symptomatic hypocalcemia. Am J Surg 2009;197 (5):648-54.

[5] Lombardi CP, Raffaelli M, Princi P, et al. Early prediction of post thyroidectomy hypocalcemia by one single iPTH measurement. Surgery 2004;136 (6):1236-41.

[6] Quiros RM, Pesce CE, Wilhelm SM, et al. Intraoperative parathyroid harmone levels in thyroid surgery are predictive of postoperative hypoparathyroidism and need for vitamin D supplementation. Am J Surg 2005;189 (3):306-9.

[7] Proye C, Carnaille B, Maynou C, et al. The parathyroid risk in thyroid surgery. Argument against the early postoperative prescription of vitamin D. Experience with 729 thyroidectomies in 1988. Chirurgie 1990;116 (67):493-500.

[8] Mulholland MW, Doherty GM. Complications in surgery. $2^{\text {nd }}$ edn. Philadelphia, PA: Lippincott Williams and Wilkins 2006: p. 553-6.

[9] Payne RJ, Hier MP, Tamilia M, et al. Same-day discharge after total thyroidectomy: the value of 6-hour serum parathyroid hormone and calcium levels. Head Neck 2005;27 (1):1-7. 